\title{
MODIFIED HEMI-FONTAN PROCEDURE ON THE BEATING HEART
}

Franz-Xaver Schmid, MD, Friedrich Wippermann, MD, Christoph Kampmann, MD, Michael Hilker, MD, and Hellmut Oelert, MD, Mainz, Germany

Indication, timing, and type of surgical intervention in patients with single ventricle physiologic condition are a continuing challenge. For patients who have significant risk factors for a poor Fontan outcome, the bidirectional cavopulmonary anastomosis has been shown to be an effective staging procedure by providing pulmonary blood flow without volume loading of the single ventricle. ${ }^{1,2}$ It offers the benefit of a relatively simple surgical approach and can be performed as a closed procedure with the heart beating on partial cardiopulmonary bypass. However, later completion of the Fontan circulation can only be accomplished by extensive dissection of the cardiac end of the superior vena cava and of the right atrium. Additionally, there may be an increased risk for arrhythmias and phrenic nerve palsy. Therefore the socalled hemi-Fontan procedure, including an anastomosis between both ends of the divided superior vena cava and the pulmonary artery in conjunction with an intraatrial patch occlusion of the cavoatrial junction, was introduced. ${ }^{3}$ This procedure involves opening of the right atrium during a short period of aortic crossclamping and cardioplegic arrest and

From the Departments of Cardiothoracic Surgery and Pediatric Cardiology, Johannes Gutenberg-University Hospitals, Mainz, Germany.

Received for publication July 16, 1998; accepted for publication July 30, 1998

Address for reprints: Franz-Xaver Schmid, MD, Department of Cardiothoracic Surgery, University Hospitals, Langenbeckstrasse 1, D-55131 Mainz, Germany.

J Thorac Cardiovasc Surg 1999;117:188-90

Copyright (๑) 1999 by Mosby, Inc.

$0022-5223 / 99 \$ 8.00+0 \quad \mathbf{1 2 / 5 4 / 9 3 6 1 6}$

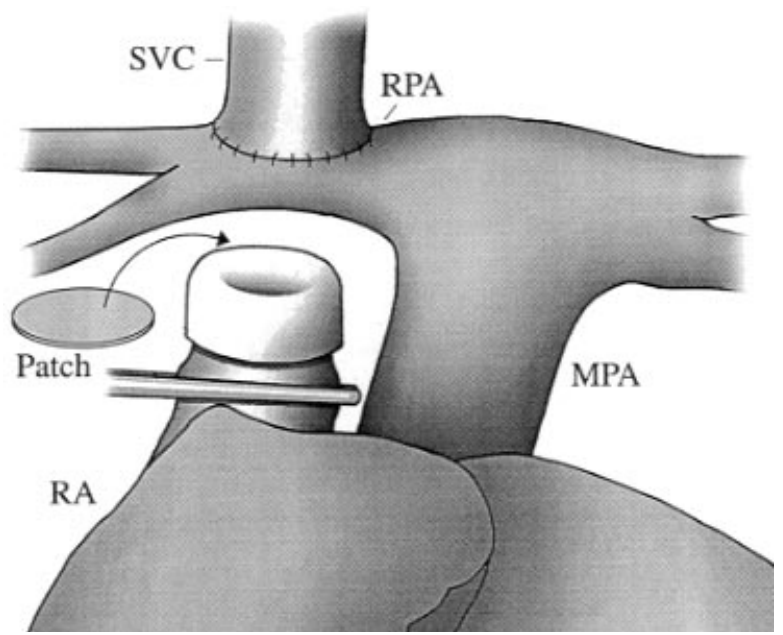

Fig 1. Schematic display of the operative procedure: Proximally eversion is facilitated by dissection and mobilization of the full length of the superior vena cava $(S V C)$. RPA, Right pulmonary artery; $M P A$, main pulmonary artery; $R A$, right atrium.

may increase the overall risk of the operation. We developed an alternative technique for a hemi-Fontan procedure on cardiopulmonary bypass without the requirement for aortic crossclamping.

Technique. The operation is performed through a median sternotomy incision. Cardiopulmonary bypass is established by aortic perfusion and drainage via cannulation of the right atrium and the innominate vein. Clamps are placed on the 
superior vena cava with some distance superior to the cavoatrial junction and just below the innominate vein orifice. The superior vena cava is transected at the level of the superior margin of the right pulmonary artery. The cut edges of the cardiac end of the divided superior vena cava are everted with gentle axial traction. The venous stump is kept everted until a patch is sutured within the superior vena cava near its entrance site to the right atrium (Fig 1). After complete internal occlusion, the eversion is reduced. The operation is completed with the creation of a total cavopulmonary connection with anastomosis of the cranial and cardiac end of the divided superior vena cava into a longitudinal incision in the superior and inferior aspect of the right pulmonary artery. Thus a hemi-Fontan circulation is established (Fig 2). Subsequent completion of the Fontan procedure requires the excision of the patch from within the right atrium and construction of the lateral atrial tunnel.

Patients and results. From June 1, 1996, to April 30, 1998, 14 infants with hypoplastic left heart syndrome who survived the Norwood procedure (including pulmonary artery to ascending aorta anastomosis with homograft augmentation of the aortic arch, atrial septectomy, and placement of a 3.5-mm modified right Blalock-Taussig shunt) underwent a modified hemi-Fontan operation as an intermediate stage for an eventual Fontan procedure at our institution. The hemi-Fontan operation was performed at a mean age of $7.6 \pm 5.4$ months (range, 3 to 14 months). Mean bypass time was 68.9 minutes (range, 40 to 127 minutes). All surgical procedures were performed while the heart was beating. Oxygen saturation after discontinuation of cardiopulmonary bypass was $83.1 \%(75 \%$ to $88 \%$ ). There was no operative death. The early postoperative course was smooth in all patients, with weaning from the ventilator and discharge to the ward between 2 and 6 days after the operation. The youngest patient in this series had significant pleural effusions and required chest tube drainage for 5 days. Chylothorax occurred in one patient.

Three weeks of a fat-free diet was required for cure. No arrhythmias occurred, and no antiarrhythmic medication became necessary.

After a period of 8, 12, and 13 months of follow-up in 3 patients, the hemi-Fontan circulation was converted to a complete Fontan circulation by placement of an intra-atrial baffle. During the short follow-up period, no complications occurred. All patients are alive and well.

Comment. The hemi-Fontan operation (functional bidirectional cavopulmonary anastomosis) has proved to be a safe and effective preparatory stage for a Fontan procedure, especially in young and high-risk candidates. ${ }^{4}$ The technical modification that is reported here offers several potential advantages. The total cavopulmonary anastomosis can be performed on normothermic cardiopulmonary bypass with the heart beating. Avoiding myocardial ischemia may be of substantial importance in patients with increased surgical risk, as are patients experiencing second-stage palliation of hypoplastic left heart syndrome. ${ }^{5}$ The absence of intra-atrial suture lines by performing an extracardiac occlusion of the superior vena

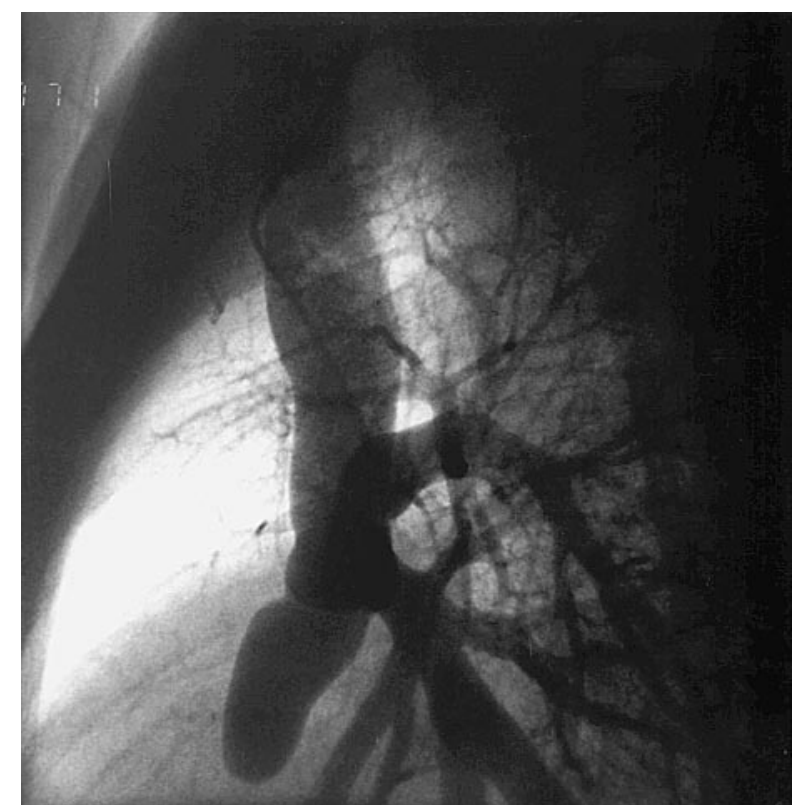

Fig 2. Lateral angiogram reveals wide patency of the cavopulmonary anastomoses and closure of the cavoatrial junction. The angiogram was performed during follow-up study before Fontan completion. Bidirectional pulmonary blood flow is noted.

cava at its right atrial junction and the avoidance of extensive dissection at the time of the Fontan completion may contribute to decrease the incidence of atrial arrhythmias. Moreover, creation of the inferior cavopulmonary anastomosis is facilitated because it can be performed when the clamp at the cardiac end is already released.

An adequate length of the cardiac end of the superior vena cava is critical for its eversion. Ligation and division of small venous branches draining to the superior vena cava and of the azygos vein allow extensive mobilization of the superior vena cava. Azygos continuation of the inferior vena cava may significantly reduce the length of the vein available for the anastomosis. Thus the vascular clamp has to be placed near the cavoatrial junction, with the sinus node at risk for damage. In this situation we do not recommend our modified surgical procedure.

In patients with an additional left superior vena cava, this also is anastomosed to the pulmonary artery in an end-to-side fashion but is oversewn at its cardiac end. Moreover, ensuing completion of the Fontan circulation after the aforementioned hemi-Fontan modification can also be performed easily as a total extracardiac cavopulmonary procedure.

In conclusion, the hemi-Fontan operation can be accomplished without cardiac arrest and low operative morbidity and mortality in the setting of hypoplastic left heart syndrome and in most patients with a functional single ventricle. Longterm follow-up of this technique will provide further information on its potential advantages. 


\section{REFERENCES}

1. Albanese SB, Carotti A, Di Donato RM, et al. Bidirectional cavopulmonary anastomosis in patients under two years of age. J Thorac Cardiovasc Surg 1992;104:904-9.

2. Hopkins RA, Armstrong BE, Serwer GA, et al. Physiologic rationale for a bidirectional cavopulmonary shunt. J Thorac Cardiovasc Surg 1985;90:191-8.
3. Jacobs ML, Rychik J, Rome JJ, et al. Early reduction of the volume work of the single ventricle: the hemi-Fontan operation. Ann Thorac Surg 1996;62:456-62.

4. Lamberti JJ, Spicer RL, Waldman JD, et al. The bidirectional cavopulmonary shunt. J Thorac Cardiovasc Surg 1990;100:22-30.

5. Jonas JA. Intermediate procedures after first stage Norwood operation facilitate subsequent repair. Ann Thorac Surg 1991;52:696-700. 